\title{
MOVIMIENTOS BARRIALES: REIVINDICACIÓN DEL DERECHO A LA CIUDAD
}

\author{
Neighborhood Movements: Acceptance of the Right to the City
}

Movimentos nos bairros: reivindicação do direito à cidade

Cindy Riguey Cuéllar Obando (Colombia)

Universidad Federal Fluminense

Maestría en Política Social

chin157@hotmail.com

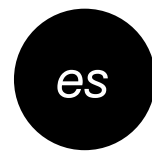

\section{RESUMEN}

Este artículo de revisión explora la problemática de las diferentes formas de reivindicación de los habitantes de barrios populares de Bogotá a finales de la década de 1970 e inicios de la década de 1990. Se presta especial atención a los procesos culturales y políticos de la coyuntura que define los medios, las modalidades de las protestas y el espacio en el que fueron realizadas. A partir de este escenario, se analiza el desarrollo urbano en ese periodo en Bogotá, en el que se encontró un crecimiento fragmentado y acelerado, sobre todo en las ocupaciones lejanas y desconectadas y, en segundo lugar, en la situación marginal de los suburbios y el densamente poblado centro de la ciudad, lo cual condujo a respuestas erróneas por parte del Estado y la Administración distrital, mientras que los habitantes de los suburbios y las ocupaciones urbanas desarrollaron iniciativas para reclamar su derecho a la ciudad y cambios en la política urbana, con lo cual se creó un proceso continuo de construcción de una identidad colectiva.

PALABRAS CLAVE: movimientos sociales, reivindicación social, organizaciones comunitarias, movimientos sociales, movimientos barriales.

\section{ABSTRACT}

This article explores the issue of the acceptance of people who lived in popular neighborhoods in Bogota in the late 70s and at the beginning of the $90 \mathrm{~s}$. We highlight the cultural and political processes of the circumstances that defined the media, the protests, and the space where they occurred. From this scenario, we analyzed the urban development of Bogotá during this period, finding a fragmented and accelerated growth in distant and disconnected occupations. We also found a marginal situation in the suburbs and in downtown Bogotá. This led to wrong answers from the State and the district's administration while the inhabitants of these sectors protested in order to claim for their right to the city and to generate changes within the urban policy. All this helped create a collective identity.

KEYWORDS: social movement, social acceptance, community organizations, social movements, neighborhood movements.

PARA CITAR ESTE ARTÍCULO/TO CITE THIS ARTICLE/PARA CITAR ESTE ARTIGO: Cuéllar Obando, C. R. (2015). Movimientos barriales: reivindicación del derecho a la ciudad. Panorama, 9 (16), 66-76. 
INTRODUCCIÓN

A partir de las grandes migraciones de población campesina a la ciudad, empieza a ser notable una problemática urbana que parte de la industrialización y los nuevos pobladores en las ciudades. Así es como no se pueden ignorar las nuevas distinciones de clase que surgen a partir de este proceso, es decir que el campesino pasó a ser obrero, el burgués pasó a ser patrón o industrial. Como parte de esta dinámica industrial, en la ciudad se presenta un cambio morfológico, evidenciado a partir de la formación de tugurios, barrios populares y barrios ilegales, cuyos habitantes eran los nuevos ciudadanos migrantes o aquellos con pocos recursos.

Sin embargo, los procesos derivados de la industrialización, como lo fueron la marginalidad y un crecimiento urbano fragmentado, tienen implícitos los actores urbanos y su papel en la transformación de la ciudad. Teniendo en cuenta que después de la década de 1950 surgen los nuevos movimientos sociales, y estos nuevos actores no son solo obreros o campesinos, sino que tienen una nueva característica: son urbanos y de las clases populares.

Las dinámicas urbanas en Bogotá durante la década de 1970 hasta principios de la década de 1990 son el fondo del surgimiento de los movimientos populares urbanos. En este periodo, se empezaron a vislumbrar las identidades colectivas, culturas y lenguajes urbanos. Además, se abrieron espacios para la asociación y participación política de los pobladores como herramienta para el fortalecimiento de la democracia, dando a los nuevos movimientos sociales el papel de constructor de ciudadanía.

Desde una perspectiva marxista, la industrialización genera un nuevo estilo de vida urbana con contradicciones, que producen conflictos en el ámbito urbano y entre el Estado y otros actores urbanos.

La forma que asume la organización social del espacio, la estructura interna y las contradicciones que se presentan dentro de un sistema urbano son la expresión del modo de producción históricamente dominante, con sus correspondientes relaciones económicas, sociales e ideológicas de producción y reproducción (Grupo de Estudios José Raimundo Russi, 1975, pp. 10-11).

Este modo de producción se manifiesta con el surgimiento de una nueva clase con una posición social estructuralmente inestable, debido a que no está inmersa aún en el sistema económico. En segundo lugar, se evidencia en el ordenamiento espacial de la ciudad como zonas deterioradas y aisladas, llamadas urbanizaciones espontáneas, que son aquellas causadas por las urbanizaciones piratas en la periferia de la ciudad.

Entre estas contradicciones, se desarrollan los nuevos movimientos sociales, los cuales se caracterizan por poseer una base social definida, una organización, una continuidad y una fuerza para transformar las estructuras (Castells, 2012, p. 218). Sin embargo, se pueden presentar organizaciones o asociaciones vinculadas más a dinámicas específicas en locaciones específicas, que pueden ser nombradas como movimientos barriales ${ }^{1}$, encargados de fortalecer el tejido social barrial, en busca de tener autonomía del Estado y los partidos políticos, con la autogestión, e impulsar proyectos para el beneficio del barrio mismo.

Las organizaciones y asociaciones que buscan la reivindicación del derecho a la ciudad, concepto con el cual Lefebvre (1976, p. 85) engloba una serie de derechos que son indispensables para los habitantes de las ciudades: el derecho a la actividad participante y el derecho a la apropiación que no es el mismo que el de la propiedad, con lo cual se convierte a la ciudad en un espacio de encuentro, convivencia y disfrute.

Bogotá, al ser la ciudad capital, se convirtió en el principal escenario de las nuevas dinámicas sociales, políticas

1 Los movimientos barriales son definidos como o una gama de nuevas formas de lucha y de resistencia cotidiana. Su amplitud marca la respuesta a la supresión sistemática de los intereses vitales de todos los sectores populares en beneficio de una reducida minoría, y en algunos países alcanza una envergadura que consigue arrancar sustanciales concesiones a las dictaduras - abiertas o disfrazadas - establecidas ahí. La expresión organizativa de esta resistencia es todavía muy dispar: varía con la coyuntura económica y política, puede aparecer como muy aislada e inoperante durante largas fases para de repente confluir en amplias corrientes de oposición, aprovechando posibles fisuras en el sistema dominante. Buena parte de estas nuevas experiencias de lucha se dan a partir del contexto barrial, muchas veces en los barrios pobres e improvisados que llegaron a servir de morada para millones de familias trabajadoras latinoamericanas. En muchos casos, estas luchas por la vivienda, el agua y la electricidad ya no se dan en forma aislada, sino que van estableciendo entre sí una red de intercambio de experiencias y de organización que puede llegar a convertirse en movimientos barriales. Dentro de estos movimientos, se desarrollan nuevas formas de ayuda mutua y de aprendizaje político común en consejos vecinales, comunidades eclesiales de base, grupos femeninos, ollas comunes y talleres o, en versión negativa, como criminalidad colectiva por parte de bandas de jóvenes desocupados (Evers, Müller-Plantenberg y Spessart, 1982, p. 705).
Movimientos

barriales:

reivindicación

del derecho a la ciudad

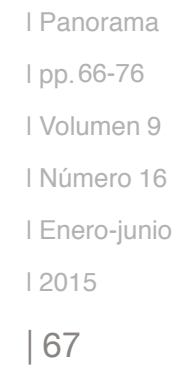

I Panorama

Volumen 9

Numero 16 
Cindy Riguey Cuéllar Obando I

\section{actúan?}

Para responder a la inquietud, se busca, en primer lugar, comprender el proceso de urbanización en Bogotá, caracterizado por experiencias de resistencia y movilización, pero también por la intervención del Estado para la promoción de la democracia. En segundo lugar, se desean reconocer las diferentes maneras de estructurar y concebir las organizaciones populares como los mediadores entre el Estado y los habitantes. En tercer lugar, caracterizar los comportamientos de la protesta y movilización urbana en Bogotá. Por último, estudiar cómo estas organizaciones han contribuido culturalmente y en la construcción de tejido social en estos asentamientos.

Finalmente, se hace un balance de los diferentes movimientos urbanos populares, teniendo en cuenta los objetivos y su actuación para la consecución de sus metas. Siendo un desafío conseguir la participación de los nuevos actores populares urbanos tanto para la formación de las organizaciones como para demandar la ayuda al Estado para suplir necesidades que el entorno urbano estaba generando en este tipo de asentamientos.

\section{EL CRECIMIENTO DE LA CIUDAD}

Desde la Colonia, Bogotá es la capital de Colombia, donde se han desarrollado la mayoría de las actividades comerciales, es el centro de las principales instituciones de Gobierno Nacional, además de ser el centro administrativo y comercial, es la ciudad con mayor población en el país. A partir de su fundación, Bogotá siguió un lineamiento de crecimiento, con estrictas normas sobre la construcción y la posesión de terrenos. Durante varias décadas, se conservaron características de la vida rural en un entorno con elementos urbanos (Lemus, 2009, p. 50). A lo anterior se suma el periodo de guerras civiles que dificultaba la expansión de la ciudad, por lo que se prefería rentar los predios ya existentes que levantar nuevas construcciones.
Sin embargo, este crecimiento controlado tuvo un fuerte giro después de la década de 1950 con el proceso de industrialización: "los efectos perversos de una modernización capitalista que a la vez expulsaba a los campesinos de las zonas rurales, los atraía como mano de obra hacia los centros urbanos con la ilusión de seguridad y progreso" (Torres, 2007, p. 17), con lo cual se generó un rápido crecimiento demográfico y urbano de Bogotá.

\section{EXPANSIÓN URBANA 1950-1989}

A finales de la década de 1940 y principios de la década de 1950, la industria representó la mayor fuente de trabajo en la ciudad, e impulsó el crecimiento urbano debido a la migración del campo a la ciudad. A este fenómeno económico se suman los desplazamientos causados por la violencia, consecuencia de los enfrentamientos entre cuadrillas de liberales y conservadores (Maya, 2007, p. 31). Este fenómeno permitió ver en la ciudad una diferenciación entre barrios residenciales y barrios de obreros, estos últimos ubicados en su mayoría en el sur y suroeste de la ciudad, lo cual dio origen a las construcciones ilegales.

\section{Durante la década de 1950, la ciudad fue objeto de grandes cambios. Para 1951,Bogotá tenía 700000 habitantes aproximadamente; los problemas sociales y la necesidad de vivienda crecieron descomunalmente, los programas y las acciones emprendidas hasta ese momento resultaron insuficientes frente a la gran demanda, siguieron formándose barrios clandestinos ocupando, en condiciones de riesgo, la periferia urbana (Maya, 2007, p. 35).}

El proceso de consecución de un espacio para vivir por parte de estos migrantes y ciudadanos que no tenían los medios para adquirir una vivienda fue principalmente con la urbanización pirata.

$$
\begin{aligned}
& \text { Las condiciones específicas en Bogotá } \\
& \text { estarían determinadas aparentemente } \\
& \text { porque los procesos de ocupación informal } \\
& \text { del suelo se han dado en forma pausada y } \\
& \text { relativamente pacífica si se compara con } \\
& \text { otras ciudades, pues la invasión, el conflicto } \\
& \text { y el enfrentamiento con ciertos niveles de } \\
& \text { la violencia no han sido la pauta en Bogotá } \\
& \text { (Camargo y Hurtado, 2013, p. 83). }
\end{aligned}
$$

Este tipo de urbanización permitió que muchos accedieran a vivienda propia, que fue creciendo desordenadamente 
por la zona sur de la ciudad, sobre terrenos en situación de riesgo, ya fuera por deslizamiento, ya fuera por inundación, lejos de los servicios básicos, como las escuelas, medios de transporte público, hospitales, agua potable y energía eléctrica. La adquisición de terrenos a bajo precio se empezó a popularizar, cuyos terrenos eran ofrecidos por vendedores no autorizados que desacataban los lineamientos de urbanización establecidos en la época. Estos lineamientos establecían unos mínimos sobre el entorno, las vías de acceso y los servicios domiciliarios ${ }^{2}$. Sin embargo, las diferentes normas y multas que se decretaron para limitar el crecimiento de barrios ilegales no fueron suficientes para detener el acelerado desarrollo de estos asentamientos.

La intervención del Gobierno en el tema de vivienda en este periodo fue con la oferta de construcción de vivienda a bajo costo; proyectos financiados por el Instituto de Crédito Territorial (ICT) y actores privados, como la Unidad de Poder Adquisitivo (UPAC) y las corporaciones de ahorro y vivienda (CAV). Sin embargo, la financiación no fue suficiente para enfrentar la creciente expansión de los asentamientos irregulares, de forma que el Departamento Administrativo de Planeación Distrital (DAPD) redefinió sus lineamientos (Corporación Sur, 2003, p. 45). En primer lugar, propuso la ampliación del perímetro urbano de la ciudad, de este modo se prestarían servicios básicos en algunos asentamientos alejados del centro de la ciudad, haciendo parte de las zonas urbanizables. A pesar de establecer el perímetro urbano, esta restricción no tuvo éxito para contener la expansión de los asentamientos irregulares, por lo cual se hizo necesario integrarlos al tejido urbano de la ciudad, así violaran la normativa de planeación urbana.

El Gobierno, al ver que era imposible detener la vivienda ilegal, decidió llevar a cabo programas que ayudaran a la seguridad y el saneamiento de los asentamientos ya existentes o en proceso de construcción. Uno de ellos fue el programa de autoconstrucción en 1963, 1967 y 1971. La Administración se encargó de aportar los lotes y una parte de los materiales para que los beneficiados construyeran su propia vivienda bajo la supervisión y asesoría de expertos en edificación, que fueron suministrados por la Alcaldía (Torres, 1984, p. 135). En

2 El Acuerdo 30 de 1961, en lo que concierne a la fijación de las especificaciones de construcción, control en la ejecución de las obras y recepción de ellas y de la Personería Distrital sobre la presentación de garantías y legislación de contratos que se exijan a los urbanizadores y a los propietarios de las tierras. De igual manera, estableció las áreas que se destinaban a uso público para que las urbanizaciones tuvieran vías, parques y zonas escolares. segundo lugar, se optó por la erradicación de algunos barrios. En Bogotá se destaca el desalojo del barrio Policarpa Salavarrieta ubicado en el sur de la ciudad, en el que participó el Ejército y la Policía. Sin embargo, este tipo de medidas fueron abandonadas al surgir el movimiento de los destechados ${ }^{3}$ en la década de 1970, que pedían una intervención adecuada del Estado en lo que respecta a la vivienda de interés social, integración de los barrios periféricos a la ciudad y la cobertura de servicios de recolección de basura, acueducto y alcantarillado y energía eléctrica (Torres, 1984, p. 135). De esta forma, el Gobierno optó por llevar a cabo programas de rehabilitación de los asentamientos, con los decretos 1025 de 1987 y 067 de 1988, que señalaban las zonas de rehabilitación, además de permitir el proceso de densificación de la ciudad.

El crecimiento de los barrios ilegales, los asentamientos espontáneos y la demanda de una política habitacional eficaz obligaron al Gobierno a replantear sus políticas enfocadas al desarrollo urbano. Ya no se trataba de grandes obras de infraestructura para hacer parecer moderna la ciudad, sino que se debía centrar en acciones sobre los barrios y la expansión desordenada de aquella, garantizar la salubridad y la prestación de servicios. Estos primeros intentos de solucionar los problemas de hábitat y pobreza permitieron desarrollar en los próximos años políticas multidisciplinarias, que no solo incluían los esfuerzos del Estado por brindar vivienda a quien lo necesitara, sino que se debía incidir positivamente sobre la calidad de vida de la población en situación de pobreza, lo cual implicaba ir más allá de una intervención físico-espacial.

\section{DÉCADA DE 1990}

Para finales de la década de 1980 y comienzos de la década de 1990, en Colombia se presentó un aumento en la violencia entre guerrillas con fuerte presencia en el campo, tras lo cual esta se comenzó a infiltrar en las ciudades bajo el título de milicias o pandillas urbanas. El conflicto armado interno trae consigo un nuevo mercado ilegal que es el de las drogas, con lo cual se abre campo en la economía tanto rural como citadina. Así es como muchos de los barrios marginales ya existentes fueron influidos por un sentimiento de inseguridad, por lo que se generó desplazamiento dentro de la misma ciudad

3 Se realizó un paro cívico nacional en 1977, que reivindicaba el derecho al techo, servicios públicos y equipamientos en los barrios obreros (Torres, 1984, p. 135). $\mid \begin{aligned} & \text { Movimientos } \\ & \text { barriales: } \\ & \text { reivindicación }\end{aligned}$ del derecho a la ciudad . 
(Chaparro, 2002, p. 58). Por otro lado, aún persistía el desplazamiento forzado debido al conflicto; en estos años, fue masiva la migración de campesinos a la ciudad y, como en los periodos anteriores, esta fue incapaz de responder a las demandas de estos nuevos pobladores. Es decir que para la década de 1990 la población de Bogotá era de casi $6^{4}$ millones de habitantes, la población se duplicó en dos décadas, presentando una tasa de crecimiento anual de $2.92 \%$ (Chaparro, 2002, p. 59). Por esta razón, se empezaron a reproducir nuevas formas de construcción de vivienda en la capital. De 1964 a 1973, la autoconstrucción superaba a cualquier otra forma de producción (capitalista, encargo, estatal) en $42.9 \%$ sobre el total de viviendas. Al igual que de 1973 a 1985 cuando la autoconstrucción representaba $49.9 \%$, con una baja producción del Estado, 19.5 \% (Alfonso, Hataya y Jaramillo, 1997, p. 55).

La respuesta del Gobierno en este caso fueron los programas de mejoramiento integral que evolucionaron con las distintas administraciones para legalizar los barrios y mejorar las condiciones de vida de los moradores. Sin embargo, la novedad en estos programas fue la creación de vínculos sociales y la cooperación entre la población objetivo y los entes del Gobierno, además se involucró a la sociedad organizada (Torres, 2009, p. 28). Este tipo de participación permite visualizar, en los diferentes resultados arrojados por las evaluaciones de los programas, que es la herramienta para responsabilizar a la comunidad por su entorno y garantizar la sustentabilidad.

Cuando la localización de estos barrios
no sea incompatible con los planes de
desarrollo urbano, se procederá dentro de las
condiciones anteriores, prestando especial
atención a la titulación de tierras en aquellos
casos en que no estuviese saneada. En cuanto
a la erradicación se menciona: "En los casos
donde la urbanización espontánea sea
incompatible con los Planes de Desarrollo
Urbano, se procederá a su relocalización
dentro del sistema de prevención de
Tugurios (Conpes 3604,2009 , art. 4).

Volumen 91 Número 16 | Enero-junio I 2015 I Entre las estrategias desarrolladas por la Alcaldía Mayor de Bogotá, se identifica el Proyecto Sur con Bogotá. Este sector de la ciudad es de gran importancia, puesto que concentra la mayoría de los asentamientos informarles y al mismo tiempo la mayoría de la población en situación de pobreza y desigualdad. Sus principales

4 La población en 1993 era de 5484244 habitantes. objetivos fueron: 1) mejorar las condiciones ambientales y urbanas estableciendo sistemas adecuados de operación y mantenimiento; 2) consolidar procesos sociales para lograr comunidades capaces de asumir y gestionar la solución de sus problemas y la ejecución de los proyectos, y 3) diseñar e implementar mecanismos de coordinación interinstitucional que aseguren la reciprocidad del proyecto (Torres, 2009, p. 65). Para la ejecución del proyecto, se requirió la coordinación de los actores y las instituciones, además de integrar el componente de desarrollo social, que suponía la participación de las organizaciones comunitarias para la toma de decisiones sobre la planeación y gestión del proyecto. El proyecto incluyó como eje transversal el fortalecimiento del tejido social, debido a los conflictos sociales y la violencia característica de la zona.

\section{POBLADORES POPULARES URBANOS Y LUCHAS URBANAS}

La violencia y la oferta de trabajo y mejor calidad de vida en la ciudad causó en el paisaje urbano la llegada de millones de habitantes que demandaban un espacio para establecerse a lo cual se suman demandas de vivienda y equipamiento de servicios básicos. Las respuestas insuficientes del Gobierno para atender las necesidades de los nuevos pobladores populares tuvieron como resultado múltiples manifestaciones y protestas, la primera y pionera fue en 1977, que fue el primer paro cívico nacional, de ahí en adelante entre el 1971 y 1985 se realizaron 285 paros cívicos y 416 otras actividades para expresar el descontento (López, 1987, p. 15).

Frente a las respuestas no acertadas, los migrantes que no se vinculaban como obreros empezaron a trabajar en actividades de la economía informal. De forma que las limitaciones económicas se convirtieron también en limitaciones para vincularse a la dinámica de la ciudad, y por lo tanto se dificultó la obtención de vivienda. Esta dinámica compartida entre los moradores de un mismo asentamiento permitió la construcción de lazos de sociabilidad para la búsqueda y materialización de sus objetivos.

En Bogotá en medio de una coyuntura inflacionaria (entre 1970 y 1974 el costo de vida aumentó en $120 \%$ ), de surgimiento de nuevos grupos de izquierda, de ascenso de otras luchas sociales y radicalizaciones de 
sectores de la Iglesia católica, nuevas generaciones de pobladores populares plantearon sus demandas sociales (principalmente vías, transporte y escuelas) por métodos diferentes de los empleados por los viejos dirigentes comunales. En contraste con las décadas anteriores, se generalizaron formas manifiestas de protesta (marchas, mítines, plantones y paros cívicos) en demanda de estos servicios públicos (Torres, 2007, p. 27).

La dinámica de ocupación ilegal conlleva los asentamientos a carecer de dinámicas de ordenamiento y planificación formal, teniendo como resultado falta de infraestructura urbana, como calles, parques, servicios públicos, además de la falta de presencia de entes reguladores, con lo cual se permite que los pobladores establezcan los patrones de ocupación y liderazgo en el territorio.

Después de la autoconstrucción de la vivienda, la mayor preocupación en estos asentamientos fue la consecución de agua, energía eléctrica, transporte, alcantarillado y espacios de encuentro. Estas necesidades comunes permitieron la asociación de moradores, a fin de llevar a cabo acciones puntuales para la resolución de estas necesidades.

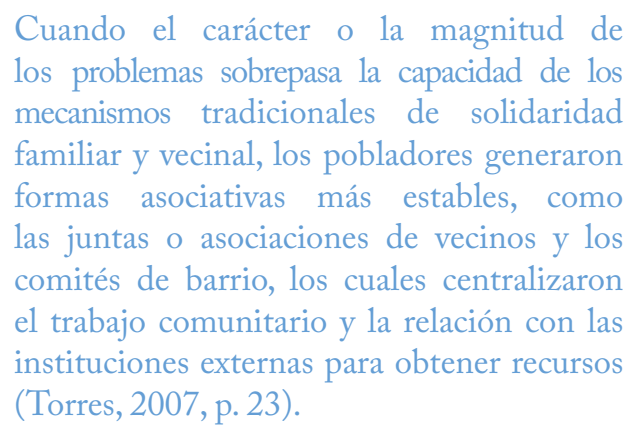

Este autor define como

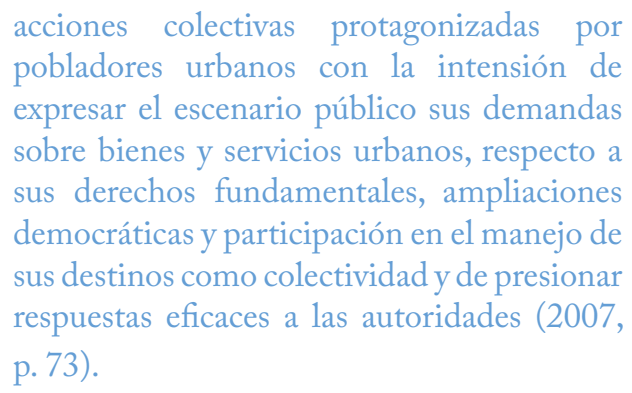

Es posible reconocer cuatro estrategias de organización o relaciones de los pobladores, las cuales muestran las formas de asociación como mecanismo de participación.
Como se dijo, el primer paso para promover estos asociacionismos en Bogotá fue el paro cívico nacional de 1977, en el cual se expresaron las clases subordinadas de la ciudad: obreros, habitantes de los barrios populares, empleados públicos, vendedores ambulantes y jóvenes de barrios populares. Esta expresión fue diferente de las otras marchas y movilizaciones, debido a que tuvo lugar la paralización total o casi total de las actividades de la ciudad para exigir al Gobierno una solución a los problemas relacionados con los servicios públicos, los cuales afectan a la mayor parte de la población.

Para la misma época (la década de 1970, incluso la década de 1980), se llevó a cabo la iniciativa de la Administración distrital para construir la avenida en los cerros orientales de la ciudad, sin embargo, junto a esta modernización de las vías, se propuso una política para los tugurios de esta zona basada en reprimir, prevenir y corregir. En contra de la erradicación de las viviendas en barrios ilegales y el desplazamiento de las familias, surgen los comités prodefensa de la zona oriental ${ }^{5}$, que se habían organizado para la construcción comunitaria de acueductos, escuelas, jardines, salones y vías, y que habían realizado las gestiones para la legalización de los servicios públicos, y conformaron la Asociación de los Barrios Nororientales de Chapinero, organización que más tarde se juntaría con la Asociación de Vecinos Pro-Ayuda Mutua de La Casona de San Diego, cuyos miembros eran los habitantes de Paraíso, San Martín, Pardo Rubio y Mariscal Sucre (Grupo de Estudios José Raimundo Russi, 1975, p. 164).

\section{El día anterior al desalojo se encuentran ya en terrenos de La Casona un gran número de compañeros dispuestos a enfrentarse con la fuerza pública... se encuentran cerca de 200 personas reunidas... al llegar la policía, la gente en forma ordenada y pacífica, manifiesta su disposición de defender su posesión y su lucha por la vivienda de tantos años. Se apela a diversos recursos}

5 Estos comités, en un principio, no se oponían al Programa Integrado de Desarrollo Urbano de la Zona Oriental de Bogotá, que incluía en sus proyectos la construcción de la avenida, sino que reclamaban el derecho de los habitantes a una vivienda digna, así fuera en otro lugar de la ciudad en caso de que los desalojaran. Exigían que sus predios en caso de quedar afectados por la avenida les fueran comprados a precios justos y solicitaban que la negociación de los terrenos se hiciera directamente entre el alcalde mayor y los comités prodefensa y no entre los funcionarios del Instituto de Desarrollo Urbano (IDU) y cada vecino. Posteriormente, los comités van modificando y precisando más aún sus exigencias, haciendo hincapié en el derecho a vivir en el sector de forma estable, sin tener que pagar altas tarifas e impuestos y solicitando que sean resueltos, de una vez por todas, los problemas de titulación en el área. Finalmente, y debido entre otras razones a la actitud arrogante, grosera, torpe y tramposa de los funcionarios públicos, la posición de los comités prodefensa desemboca en una negativa radical a la avenida de los cerros (Memoria Barrial, 1996-1997, cap. 3).
| Movimientos barriales:

reivindicación del derecho a la ciudad 
legales pero en caso de que estos no sirvan, tendrá que hacerse la oposición violenta... la disposición de defensa de las gentes venidas de diferentes barrios es el mejor argumento, no tan legal pero convincente, para que se desista del lanzamiento... el inspector, los abogados y todo un séquito de burócratas se retiran bajo la mirada atenta y decidida de los ahora compañeros de lucha. La victoria es completa (Grupo de Estudios José Raimundo Russi, 1975, p. 164).

Lo anterior genera esperanzas entre los miembros de los comités quienes promueven las actividades regulares, como reuniones, marchas, distribución de volantes y periódicos, asambleas, actos culturales, elaboración de pliegos, etcétera.

A causa del paro nacional y otras manifestaciones menores en barrios populares, el Gobierno reaccionó estableciendo el estatuto de seguridad, que es la forma en que Misael Pastrana y Alfonso López Michelsen buscan detener mediante represión las luchas y expresiones populares y de izquierda. Julio César Turbay, en 1978, generalizó los allanamientos, detenciones arbitrarias, torturas y desapariciones de dirigentes sociales y activistas de izquierda (López, 1987, p. 13).

La limitación de los espacios de libre expresión llevó a la clandestinidad, pero también a usar otras instituciones consideradas neutrales, como la Iglesia, la educación y los medios de comunicación, lo cual dio paso al trabajo entre pobladores de barrios populares y estas instituciones para dar solución a las necesidades de los asentamientos y al mismo tiempo crear nuevos movimientos sociales urbanos. Como represión frente a estas organizaciones nacientes, el Estado decidió institucionalizar las juntas de acción comunal para tener presencia en todos los barrios de Bogotá y canalizar las iniciativas de los pobladores (Valderrama, 2004). Esta institución se convirtió en una relación de clientelismo, razón por la cual se dejó de confiar en el Estado, y al mismo tiempo se reforzó el poder de los líderes que pertenecían a las juntas de acción comunal y se desestimularon otras formas de organizaciones colectivas.
En la década de 1980, surgen nuevas organizaciones barriales $^{6}$ independientes de las juntas de acción comunal, en las que los pobladores trabajan en conjunto con actores externos, cambiando la perspectiva de acción, ya no solo el objetivo es conseguir la cobertura de servicios, sino que se empiezan a percibir nuevas necesidades, como generación de actividades productivas, reivindicativas y culturales. Estas actividades se convirtieron en las más relevantes para las mujeres cabeza de familia.

En algunos barrios, el trabajo parroquial o pastoral de algunas comunidades religiosas desembocó en grupos juveniles o en comunidades eclesiales de base, comprometidas con acciones de promoción comunitaria y organización popular. Estas nuevas experiencias asociativas, algunas impulsadas o apoyadas por organizaciones no gubernamentales, favorecieron la organización de base, la educación de sus miembros, y ampliaron las formas de gestionar sus necesidades y demandas (Torres, 2007, p. 41).

En el barrio Jerusalén, perteneciente a la localidad de Ciudad Bolívar de Bogotá, se llevaron a cabo varias acciones colectivas en defensa de los terrenos y lotes ocupados y evitar la saturación. Esta acción se emprendió en contra de los urbanizadores ilegales con los comités cívicos de Provivienda (Villalobos, 2011, p. 92). En 1993 levantaron una acción de protesta y reivindicación por el derecho al servicio público de gas natural, llevando a cabo un paro cívico que usaron también para reclamar contra algunas medidas de impuestos y ausencia en la prestación de servicios de salud y educación en el barrio.

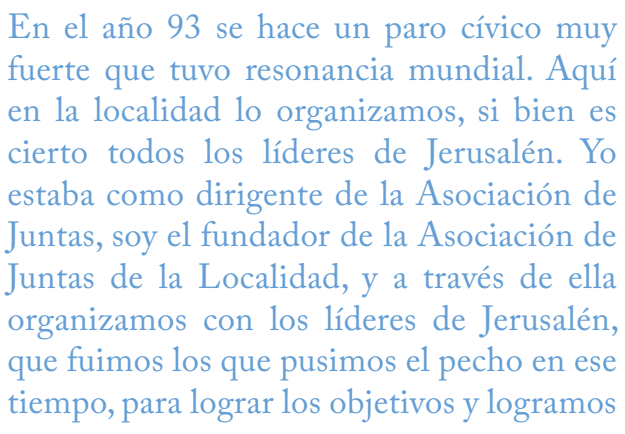

\footnotetext{
6 La historia de la mayor parte de las actuales organizaciones barriales demuestra que su origen está estrechamente ligado a la agudización momentánea de un problema muy concreto, cuya solución todos los moradores sintieron como existencial, por encima de las diferencias... las organizaciones barriales surgen con el fin de realizar tomas de terreno colectivas, o sea, antes de la formación del barrio o para defender los terrenos tomados contra el peligro del desalojo o de la demolición. También pueden ser otros problemas, desde cierto punto sentidos como insoportables para la mayoría de los pobladores (falta de luz, agua, transporte, etcétera) (Camargo, 1992, p. 45).
} 
lo que hoy en día tenemos: tener las vías; tenemos semaforización de nuestras vías; tenemos vías peatonales; tenemos gas natural, porque en ese tiempo pues era con cocinol que se hacía la comida, y logramos cambiar el cocinol por el gas natural, y a buenos precios; y lo que fue salud y educación, lo mejoramos con el paro cívico. Que si bien es cierto los compromisos que nosotros firmamos con la Administración no se cumplieron, que era como $70 \%$, logramos como un $50 \%$ que es ganancia con las comunidades (Villalobos, 2011, p. 92).

Al mismo tiempo que estas nuevas organizaciones de pobladores, aumentó el número de acciones de protesta entre 1977 y 1990, casi 259 luchas que incluyeron marchas dentro de los barrios, bloqueo de las vías principales en el sur de la ciudad, toma de las plazas representativas, etcétera. Es el caso del paro cívico local llevado a cabo en Ciudad Bolívar por la organización Instituto Cerros del Sur (García, 1991, p. 89), que exigía la protección de los derechos humanos, y sobre todo del respeto por la vida y la garantía a una vida digna. Se presentó un pliego de peticiones en el cual se pronunciaban frente a las normas y leyes ya existentes que estaban atropellando a la población.

Junto a esta movilización, se pueden nombrar otras, como la de 1985 que reclamaba por la insalubridad, con un brote de fiebre tifoidea, que para 1986 se tornó en alerta roja debido a una inundación (López, 1987, p. 12). De modo que los pobladores se tomaron la avenida de Abastos para exigir una solución definitiva a la empresa de acueducto y a la Alcaldía de Bogotá.

El 11 de octubre de 1993 se lleva a cabo otro paro cívico zonal, que fue una de las pocas movilizaciones que convocó a los medios de comunicación y a la integración de diversos sectores de la localidad de Usme. Después de este paro, se realizaron muchos más, el más representativo fue el trancazo de la cultura, en el que los manifestantes salieron en zancos bloqueando la avenida vía Villavicencio (Torres, 2007, p. 98).

Además de los paros, se realizaron otros actos simbólicos para dar a conocer las organizaciones en los barrios y generar espacios de encuentro. Es el caso del Festival de la Cometa de 1992, cuyo fin era reivindicar los derechos de la infancia y la adolescencia, junto al cual también se establece el Festival de la Alegría que representa una semana de actividades artísticas y creativas (Torres, 2007, p. 98). Estas actividades son espacios que las organizaciones abren para vincularse con la comunidad.

Estas asociaciones y organizaciones urbanas pueden considerarse de algún modo parte o no de los nuevos movimientos urbanos. En primer lugar, porque el movimiento social se refiere a la acción colectiva que se realiza en torno a conflictos sociales con una influencia alta para transformar la situación; sin embargo, estos se caracterizan por su identidad colectiva y querer incidir en la estructura del sistema social. Castells (2012, p. 234) deja muy clara la distinción entre acción colectiva urbana y movimiento urbano; este último tiene un carácter de permanencia, con origen en un territorio popular en defensa de identidades culturales y totalmente autónomo al poder estatal. Muy diferente también de las luchas urbanas, de carácter esporádico y momentáneo, que visibilizan la inconformidad.

Sin embargo, es posible clasificar las acciones hechas en los barrios populares como movimientos barriales, ya que poseen las características de los nuevos movimientos sociales solo que aplicados a un espacio limitado, en este caso cada barrio de origen ilegal. Estas acciones, se caracterizan por la tensión entre los sistemas institucionales y entre actores temporales que revelan a la sociedad la existencia de estos problemas. Pero para ser nuevos movimientos sociales aún deben dejar de ser acciones coyunturales y dar un lugar central a la expresión simbólica (Montaño y Durigueto, 2010, p. 311).

\section{CONCLUSIONES}

Bogotá, a partir de la década de 1950, se caracterizó por el crecimiento acelerado de su población. Por un lado, aquella que podía adquirir vivienda de forma legal se incorporó rápidamente a las dinámicas de la ciudad y los barrios que habitaron ya estaban conectados con los servicios públicos que ofrecía la ciudad. Por otro, aquellas grandes migraciones hacia Bogotá causadas por la violencia o en busca de mejores condiciones de vida que las del campo terminaron en asentamientos ilegales y con condiciones precarias. Fueron asentamientos que con la informalidad obtuvieron los servicios y para mejorar su calidad de vida optaron por organizarse y llevar a cabo acciones que llamaran la atención de la Administración distrital, de forma
Movimientos

barriales:

reivindicación del derecho a la ciudad

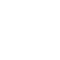


que se les reconociera como ciudadanos también y al mismo tiempo se les otorgara el derecho a la ciudad.

Los habitantes de estos barrios afrontaron la ausencia del Estado, y organizándose en juntas de acción comunal buscaron acceder a servicios públicos básicos y mejoramiento del espacio público. Esta organización propiciaba fuertes tensiones y conflictos entre los movimientos sociales y las autoridades distritales relacionados con el mejoramiento de las condiciones de vida de estas comunidades. Sin embargo, los canales del Estado no fueron los únicos medios para movilizarse y organizarse en el ámbito barrial, saliendo de la esfera electoral y jurídica, y empezando a asociarse a causa del interés comunitario, con lo cual se constituyó una participación activa dentro y en favor de la comunidad, que fortaleció el tejido social y el conocimiento de nuevos mecanismos de participación.

Aunque la postura en los planes de desarrollo nacional frente a los asentamientos informales ha variado, varios de ellos coinciden en la necesidad de atacar el problema de la informalidad en forma integral y coordinada. Específicamente, con programas para la prestación de servicios y la promoción de la participación comunitaria en zonas marginales urbanas, se plantea la necesidad de un enfoque que contemple acciones de salud, capacitación, servicios comunitarios, actividades culturales, recreativas, vivienda y servicios públicos.

El problema se encuentra en las actividades que se llevan a cabo por un tiempo corto, puesto que solo se concentran en la solución de necesidades apremiantes sin articular acciones políticas estables. Las principales tareas de las organizaciones comunitarias están relacionadas, en la etapa de formación de los asentamientos, con la autoprovisión de servicios públicos domiciliarios (agua, energía, alcantarillado). En la etapa de consolidación, dichas tareas están asociadas a la instalación de equipamientos sociales y a la provisión de programas de atención social: educación, salud, bienestar social para niños, jardines, madres gestantes, tercera edad, etcétera.

Después de que las juntas de acción comunal se desvirtuaran a causa del clientelismo, surgen nuevas formas organizativas locales, como las juntas administradoras locales o los consejos comunales que tomaron más fuerza. A su vez, las organizaciones no gubernamentales

74 | continúan siendo importantes porque apoyan procesos sociales y organizativos de las comunidades, en algunos casos en los temas de vivienda y mejoramiento barrial.

Sin embargo, estos movimientos, acciones, luchas y organizaciones en el ámbito barrial, en primer lugar, no lograron ejercer presión política suficiente para cambiar la situación, y muchas se convirtieron en acciones aisladas debido a que parte de la influencia se mide en la representación numérica. En segundo lugar, no alcanzaron una visibilidad pública, situación en la cual es el Estado el que se encarga de minimizar el problema, y lo deja marginalizado en los medios de comunicación o ignorado por completo. Por esta razón, las organizaciones barriales empiezan a trabajar en colaboración con el Estado o con partidos políticos para lograr algunos objetivos de mejoramiento de calidad de vida en el barrio.

El Estado también decidió brindar nuevos canales de comunicación en el ámbito institucional para que se hagan peticiones, solicitudes, recolección de firmas, cartas abiertas y delegaciones. Lo anterior para evitar la sensación de amenaza frente a fuertes organizaciones barriales que demandan los derechos que tienen como ciudadanos. Por lo tanto, el Estado decidió convertir las organizaciones vecinales en instituciones para promover la participación, y además hacer presencia con programas de carácter participativo ${ }^{7}$, a fin de realizar obras de mejoramiento de la calidad de vida.

La reivindicación de determinados espacios, equipamientos o servicios, así como la participación directa junto con la Administración pública en los procesos de toma de decisiones, en la financiación, en la ejecución y en el mantenimiento de los proyectos, supondrían una evolución en los procesos de recuperación de los barrios marginalizados. Dando a estos barrios y a sus habitantes identidad, cohesión social y calidad de vida.

Lo que se quiere expresar con el estímulo de la cohesión social es que se alcanzaría un trabajo conjunto y el logro de propósitos comunes que favorecerían el sentido comunitario y generarían un mayor entusiasmo

\footnotetext{
7 Este carácter participativo hace referencia a encuestas para la recolección de datos y desarrollo del diagnóstico más que incluir a la población para conocer sus necesidades, cómo ellas pueden ser una veeduría para el programa y qué nuevos aportes le darían al programa como beneficiarios. Igualmente, la participación se limita a líderes comunitarios, y deja de lado la formación de nuevos líderes dentro del barrio y la creación de espacios para el debate y la formación de lazos comunitarios.
} 
por parte de los habitantes, puesto que perciben que las cosas pueden cambiar. En cuanto a la participación, esta permite que las propuestas sean comprobadas y afinadas antes de ser adoptadas, lo cual da como resultado un mayor dinamismo en el proyecto, y en este sentido los resultados finales serán más adecuados. Por último, se esperaría que la comunidad, aun sin la iniciativa del Estado, gestione y mantenga los espacios que fueron mejorados, tras lo cual se reducen las probabilidades de abandono.

Otro punto importante en un trabajo de movimientos barriales y Estado es el fortalecimiento del asociacionismo, que ha sido clave en muchas de las reivindicaciones de mejora de los barrios. Ha servido para mediar demandas y acciones, muchas de ellas de presión que, probablemente, sin aquella no hubieran sido posibles, pero también para dar respuesta a colectivos que se encuentran en riesgo de exclusión social, tras lo cual se disminuye la vulnerabilidad con la formación y la capacitación, las redes de ayuda, etcétera.

\section{REFERENCIAS BIBLIOGRÁFICAS}

1. Acotto, L. (2006). Las organizaciones de la sociedad civil. Un camino para la construcción de la ciudadanía. En Veeduría Distrital. El control social de lo público, un derecho y deber ciudadano. Diagnóstico de las localidades de Bogotá. Localidad de Kennedy (pp.17-32). Bogotá: Veeduría Distrital.

2. Alcaldía Mayor de Bogotá (2004). Plan de Ordenamiento Territorial de Bogotá. Todas las Opiniones Cuentan. Primera revisión 2000-2003. Bogotá: Departamento Administrativo de Planeación Distrital.

3. Alfonso, O., Hataya, N. y Jaramillo, S. (1997). Organización popular y desarrollo urbano en Bogotá. Bogotá: Universidad Externado de Colombia..

4. Arango, C. (1981). Crónicas de la lucha por la vivienda en Colombia. Bogotá: Colombia Nueva.

5. Camargo, S. (1992). La construcción de la nacionalidad desde la democracia local y la trasnacionalidad de lo local: planteamientos, intuiciones y retos. Boletin Socioeconímico, 24-25, 163-182.

6. Camargo Sierra, A. P. y Hurtado Tarazona, A. (2013). Vivienda y pobreza: una relación compleja. Marco conceptual y caracterización de Bogotá. Cuadernos de Vivienda y Urbanismo, 4(8).
7. Castells, M. (2012). Redes de indignación y esperanza. Madrid: Alianza.

8. Chaparro, J. (2002). Participación y descentralización en el desarrollo local de Bogotá 1993-2002. La construcción colectiva del territorio como estrategia para incrementar la gobernabilidad de las grandes ciudades de América Latina: una aproximación al caso de Bogotá.

9. Consejo Nacional de Política Económica y Social (2009, 24 de agosto). Documento Conpes 3604. Bogotá. Recuperado de http://www.minvivienda.gov.co/Mejoramiento\%20Integral $\% 20$ de\%20Barrios/Lineamientos\%20para\%20 la\%20Consolidaci\%C3\%B3n\%20de\%201a\%20 Pol\%C3\%ADtica\%20de\%20MIB.pdf

10. Corporación Sur (2003). Proceso de planeación local. Sistematización y evaluación del proceso adelantado en 20 localidades de Bogotá, con aplicación en el Decreto 425 de 1995. En La apuesta de los ciudadanos: tercer ejercicio de planeación participativa en Bogotá. Bogotá: Fundación Corona.

11. Escalante, M. (2012). Habitabilidad en la vivienda social en edificios para población reasentada. El caso de Medellín, Colombia. EURE, 114(38), 203-227.

12. Evers, T., Müller-Plantenberg, C. y Spessart, S. (1982). Movimientos barriales y Estado. Luchas en la esfera de la reproducción en América Latina. Revista Mexicana de Sociología, 703-756.

13. García, C. (1991). Anotaciones acerca del estudio sobre los movimientos cívicos en Colombia. Medellín: Instituto de Estudios Regionales.

14. Gilbert, A. (2007). The return of the slum: does language matter? International Journal of Urban and Regional Research, 31(4), 697-713.

15. Giraldo, J. (1985). Paros y movimiento cívicos en Colombia. Bogotá: Centro de Investigacion y Educación Popular.

16. González, C. (1985). Movimientos civicos 18821984: poder local y reorganización del poder popular. Bogotá: S. D.

17. Gohn, M. D. G. (2000). O Paradigma dos novos movimentos sociais. En Teoria dos Movimentos Sociais: Paradigmas clássicos e contemporâneos (pp. 121-170). São Paulo: Edições Loyola.

18. Lefebvre, H. (1976). El derecho a la ciudad. Madrid: Península.

19. Lemus, A. y Lemus, V. D. (2009). Introducción a las infracciones urbanisticas. Bogota: Editorial Temis. 
20. López, W. (1987). La protesta urbana en Colombia. Revista Foro, 3.

21. Maya, T. (2010). Áreas residenciales y desarrollo urbano en Bogotá. Revista Urbanismos, 2, 23-55.

22. Montaño, C., y Duriguetto, M. L. (2010). Estado, classe e movimento social. São Paulo: Editora Cortez.

23. Russi, J. R. (1975). Lucha de clases por el derecho a la ciudad. Medellín.

24. Santana, P. (1986). Crisis municipal: movimientos sociales y reforma política en Colombia. Revista Foro, 1(1)

25. Santana, P. (1986). La crisis urbana y el poder local y regional: el caso colombiano. En Ciudades en conflicto. Quito: El Conejo.

26. Santana, P. (1989). Los movimientos sociales en Colombia. Bogotá: Foro Nacional por Colombia.

27. Torres, A. (2007). Identidad y politica de la acción colectiva: organizaciones populares y luchas urbanas en Bogotá, 1980-2000. Bogotá: Universidad Pedagógica Nacional.

28. Torres, C. (2009). Hábitat y vivienda: pobreza urbana y mejoramiento integral de barrios en Bogotá. Bogotá: Universidad Nacional de Colombia/Facultad de Artes.

29. Valderrama, J. C. (2004). Un siglo habitando los Cerros, vidas y milagros de vecinos en el Cerro del Cable. Text.

30. Velásquez, F. (1986). Crisis municipal y participación ciudadana en Colombia. Revista Foro, 1(1).

31. Vergel Tovar, E. (2010). Asentamientos precarios: una aproximación para su mejoramiento integral y prevención. DEARO: Revista de Arquitectura de la Universidad de los Andes, 6, 64-81.

32. Villalobos Rubiano, J.A. (s. f.). Cambios en las prácticas de la acción colectiva en las organizaciones comunitarias, debidos a la consolidación urbana en los espacios barriales de origen ilegal en Bogotá (Tesis de doctorado, Universidad Nacional de Colombia, Colombia).

Número 16 | 